\title{
Desempenho e plumagem de frangos de corte intoxicados por aflatoxinas
}

\author{
Performance and plumage of broiler chicken intoxicated by aflatoxins
}

\author{
Leandro Giacomini ${ }^{1}$ Fabiana Andréia Fick ${ }^{2}$ Paulo Dilkin ${ }^{3}$ \\ Carlos Augusto Mallmann ${ }^{4}$ Ricardo Hummes Rauber ${ }^{5}$ \\ Carlos Almeida ${ }^{3}$
}

\section{RESUMO}

O objetivo desta pesquisa foi estudar os efeitos das aflatoxinas sobre o desempenho e plumagem de frangos de corte. Foram utilizados 240 pintos machos, alimentados de 1 a 42 dias de idade com ração ad libitum sem aflatoxinas (grupo controle) e contaminada com $3 \mathrm{mg}$ de aflatoxinas $/ \mathrm{kg}$ de ração (grupo tratado) As aves foram analisadas duas vezes ao dia em relação ao seu desenvolvimento. O consumo de ração e peso dos animais foram avaliados ao final de cada fase de criação (21, 35 e 42 dias). Ao final do experimento, realizaram-se as avaliações patológicas, a pesagem das penas e a avaliação dos seus aminoácidos. As aves do grupo tratado apresentaram sinais caracteristicos de intoxicação por aflatoxinas, como desuniformidade da estatura, redução no consumo de ração e ganho de peso e alterações microscópicas e macroscópicas em órgãos e tecidos. Observou-se que o coração e o fígado das aves intoxicadas apresentaram um incremento significativo no peso médio relativo, a massa de penas foi reduzida significativamente $(33,8 \%)$ e não houve alterações na concentração e na composição dos aminoácidos, apesar do baixo desempenho dos animais intoxicados.

Palavras-chave: aflatoxinas; frangos; aminoácidos; penas.

\section{ABSTRACT}

This work had the goal to study the exposure effect of broiler chickens to aflatoxins during a period of 42 days related to its development in size and feathers. Two experimental groups of male chicks were treated with ad libitum food, one group without aflatoxins (control group) and other using contaminated food with $3 \mathrm{mg} / \mathrm{kg}$ of aflatoxins (treated group). Birds were evaluated twice a day to observe its performance and consumption of food. The weights were measured at the end of each breeding phase $(21,35$ and 42 days). At the end of the experiment animal size, weight, amount of feathers, organs and amino acids profile in blood were analyzed and compared between the two groups. The treated group showed classical signs of intoxication with aflatoxins such as less uniformity, less feed consumption and body weight gain. Also some macroscopic and microscopic organs and tissue changes were observed. Evaluation of heart and liver of the treated group showed an increase in their average relative weight. Feather mass in this group was significantly reduced (33.8\%), but its amino acids concentration was not affected despite the low performance of the intoxicated animals.

Key words: aflatoxins, broiler chickens, amino acids and feathers.

\section{INTRODUÇÃO}

As aflatoxinas são metabólitos secundários tóxicos produzidos por linhagens fúngicas do gênero Aspergillus, como A. flavus e A. parasiticus. Diversos compostos são conhecidos como aflatoxinas, porém somente as aflatoxinas $\mathrm{B}_{1}, \mathrm{~B}_{2}, \mathrm{G}_{1}$ e $\mathrm{G}_{2}$ apresentam importância toxigênica conhecida (OMS, 1983). Segundo TANAKA et al. (2001), o desenvolvimento e

${ }^{1}$ Departamento de Medicina Veterinária Preventiva, Programa de Pós-graduação em Medicina Veterinária Universidade Federal de Santa Maria (UFSM), CEP 97105-900, Santa Maria, RS, Brasil. E-mail: Leandro@lamic.ufsm.br.

${ }^{2}$ Curso de Medicina Veterinária da UFSM, CEP 97105-900, Santa Maria, RS, Brasil.

${ }^{3}$ Laboratório de Análises Micotoxicológicas, UFSM, CEP 97105-900, Santa Maria, RS, Brasil.

${ }^{4}$ Departamento de Medicina Veterinária Preventiva, UFSM, CEP 97105-900, Santa Maria, RS, Brasil. E-mail: mallmann@lamic.ufsm.br.

${ }_{5}^{5}$ Departamento de Medicina Veterinária Preventiva, Programa de Pós-graduação em Medicina Veterinária UFSM, CEP 97105900, Santa Maria, RS, Brasil. 
a formação das aflatoxinas em alimentos são dependentes de uma série de fatores, principalmente daqueles relacionados à umidade, à temperatura, ao oxigênio e à constituição do substrato.

Quando alimentos contaminados são ingeridos pelos animais, as aflatoxinas são rapidamente absorvidas, afetando principalmente o fígado, levando a distúrbios metabólitos. A degeneração gordurosa hepática e proliferação dos ductos biliares induzem diversas alterações séricas, constatadas principalmente pelo aumento da atividade de enzimas, coagulopatias e diminuição na produção de proteínas (FERNANDEZ et al., 1995; OLIVEIRA \& GERMANO, 1997). Outros órgãos como intestino, baço, linfonódos e rins também podem sofrer alterações, principalmente em animais monogástricos como aves e suínos (MARIN et al., 2002). Entre os efeitos de imunossupressão demonstrados em aves, destacam-se a aplasia do timo e da bursa de Fabricius, redução do número e da atividade das células $T$, supressão da atividade fagocitária e redução de componentes humorais, como complemento (C4), interferon e imunoglobulinas IgG e IgA(PETSKA\& BONDY, 1990; PIER, 1991; PIER, 1992; CELIK et al., 2000). Frangos alimentados com ração que contêm $300 \mathrm{mg}$ de aflatoxinas por $\mathrm{kg}$ da dieta, apresentaram diminuição dos níveis séricos de albumina e globulinas e redução da síntese protéica no fígado (GHOSH et al., 1990). A diminuição dos níveis séricos de ácido úrico, triglicerídios e colesterol também tem sido observada (HUFF et al., 1986). Os principais sinais clínicos verificados em aves intoxicadas por aflatoxinas incluem anorexia, diminuição do ganho de peso, letargia, palidez da crista, barbela e pés e sinais nervosos. DOERR et al. (1983) induziram intoxicação em frangos com $2,7 \mathrm{mg}$ de aflatoxinas $/ \mathrm{kg}$ de ração fornecida ad libitum, e não verificaram alteração significativa na quantidade de folículos de empenamento, apesar de constatar uma série de transtornos fisiológicos nos animais. Os efeitos deletérios das aflatoxinas em frangos são maiores na fase inicial de criação (até os 21 dias de vida), porém o reflexo negativo sobre o ganho de peso é persistente até a fase final de criação (HUFF et al., 1986). Assim, as aflatoxinas exercem efeitos negativos na performance avícola, comprometendo a cadeia de produção desde a diminuição de taxas de posturas de matrizes, até a redução no peso de abate dos frangos intoxicados (DOERR et al, 1983; KICHOU \& WALSER, 1993).

O presente trabalho teve como objetivo avaliar a influência das aflatoxinas no crescimento e desenvolvimento de frangos de corte, bem como efeitos sobre a formação das penas e na constituição dos seus aminoácidos.

\section{MATERIAL E MÉTODOS}

As aflatoxinas utilizadas no experimento foram produzidas pelo cultivo da cepa toxígena de Aspergillus parasiticus (NRRL 2999) em arroz, conforme metodologia desenvolvida por SHOTWEL et al. (1966). O material de cultivo foi autoclavado, secado a $50^{\circ} \mathrm{C}$ e moído. As dietas utilizadas foram isonutritivas, preparadas conforme as exigências nutricionais das fases de criação para a fase inicial (1 a 21 dias de idade); crescimento (22 a 35 dias) e final (36 a 41 dias). Os principais ingredientes utilizados foram milho, farelo de soja, vitaminas e sal mineral. As aves receberam dietas contendo $22 \%$ de proteína na fase inicial, $20 \%$ na fase de crescimento e $18 \%$ na fase final, sendo a ração formulada conforme NRC (1994). Desde a fase inicial até a fase final, as dietas foram preparadas constituindo dois tratamentos: A) 0mg de aflatoxina/ $\mathrm{kg}$ de ração (controle) e B) $3 \mathrm{mg}$ de aflatoxina $/ \mathrm{kg}$ de ração, sendo $83 \%$ de aflatoxina $B, 9,5 \%$ de aflatoxina $\mathrm{B}_{2}, 4,2 \%$ de aflatoxina $\mathrm{G}_{1}$ e $3,3 \%$ de aflatoxina $\mathrm{G}_{2}$. Após a inclusão do material de cultivo de arroz contendo as aflatoxinas na quantidade requerida no tratamento B, os níveis de aflatoxinas das dietas experimentais foram quantificados através da análise por cromatografia líquida de alta eficiência (CLAE), com limite de quantificação de $1 \mu \mathrm{g} / \mathrm{kg}$ e coeficiente de recuperação de $85,5 \%$, segundo metodologia desenvolvida e validada por MALLMANN et al. (2000).

Foram utilizados 240 pintos machos de um dia de idade, da linhagem Cobb, com peso médio de 43 $\mathrm{g}$ ao alojamento. Empregou-se um delineamento experimental fatorial casualizado com 2 tratamentos e 8 repetições de 15 aves, totalizando 16 unidades experimentais em box de 1,0 x 1,5m, alojados sobre cama de maravalha durante o experimento. Para coleta e posterior análises da constituição dos aminoácidos nas penas, foram selecionados aleatoriamente 15 frangos de cada tratamento. As aves foram alimentadas ad libitum durante todo o período experimental, exceto nos dias de pesagem, quando foram submetidas a jejum prévio de 6 horas. Adicionalmente, análises de cada lote de ração não revelaram limites detectáveis de fumonisinas $\mathrm{B}_{1}$ e $\mathrm{B}_{2}$ (DILKIN et al., 2001), toxina T-2 e deoxinivalenol (RODRIGUES, 2002), ocratoxina A e zearalenona (NURYONO et al., 2004). As aves foram inspecionadas duas vezes por dia.

A avaliação do peso das penas de todas as aves foi realizada após 42 dias de experimento. A retirada das penas das aves foi realizada após a eutanásia pelo deslocamento cervical e posterior escaldagem durante 2 minutos com água a temperatura de $60^{\circ} \mathrm{C}$. As penas foram retiradas manualmente e acondicionadas em 
embalagens individuais. Em seguida, foram secas em estufa a $50^{\circ} \mathrm{C}$ por 12 horas e pesadas individualmente. A determinação da constituição dos aminoácidos das penas foi realizada empregando CLAE conforme a metodologia desenvolvido e validada pelo laboratório decontrole de qualidade da RHODIA, observando os seguintes parâmetros para validação: limite de quantificação, limite de detecção, coeficiente de recuperação, repetibilidade e reprodutibilidade.

As necropsias foram realizadas nos animais que morreram durante o experimento e, após a eutanásia, ao término do experimento. Registraram-se as alterações macroscópicas encontradas nos órgãos e tecidos. Em seguida, coletaram-se porções de fígado, bursa de Fabricius e rins em formalina a $10 \%$ (pH de 7,8). Os fragmentos de tecidos foram processados de acordo com as técnicas histopatológicas usuais, sendo os tecidos corados com hematoxilina-eosina, de acordo com LUNA (1968). Os orgãos (fígado, bursa de Fabricius e rins) e carcaças foram coletados e pesados individualmente.

Os resultados referentes ao peso dos animais, das penas, peso das penas, e dos órgãos, assim como a concentração de aminoácidos nos diferentes tratamentos foram submetidos à análise estatística descritiva (média, desvio padrão e coeficiente de variação) e análise de variância (ANOVA). O teste de Tukey $(\mathrm{P}<0,05)$ foi aplicado para comparação das médias. Os parâmetros que não apresentaram diferença significativa quando comparados a 5\% de significância, foram também comparados à significância de $10 \%$. As análises foram realizadas empregando o programa Statgraphics, versão 5.0 (Statgraphics Manugistics, Rockville, MD, USA).

\section{RESULTADOS E DISCUSSÃO}

Os sinais clínicos observados se restringiram aos animais do tratamento $\mathrm{B}(3 \mathrm{mg}$ de Aflatoxinas $/ \mathrm{kg}$ de ração). As principais alterações foram: aglomeramento das aves nos cantos das instalações, desuniformidade na estatura, prostração, redução de consumo, apatia e palidez de crista, barbela e patas. Características semelhantes foram descritas por BONDINE \& MERTENS (1983), quando observararam alta mortalidade de aves intoxicadas com aflatoxinas, que apresentaram inclusive sinais nervosos. No nosso estudo, a mortalidade das aves intoxicadas foi evidentemente maior em relação ao grupo controle, porém a diferença não foi estatisticamente significativa.

Na tabela 1, pode-se observar que as aves intoxicadas com aflatoxinas apresentaram consumo de ração inferior em todas as fases avaliadas. Esta tendência também foi observada no ganho de peso das aves, que foi significativamente inferior ao grupo controle $(26,99 \%)$. Com relação aos resultados de conversão alimentar, observou-se uma redução significativa nas aves do tratamento B a partir da quarta semana de intoxicação. No entanto, ao final do experimento, não se observou diferença estatisticamente significativa.

Tais efeitos da intoxicação de frangos de corte por aflatoxinas podem ser minimizados utilizandose adosorventes como método preventivo. KUBENA et al. (1990) utilizaram aluninosilicatos de forma preventiva em dietas de animais intoxicados.

As aflatoxinas influenciaram significativamente sobre a massa das penas nos animais intoxicados. Ao final do experimento, as aves do grupo controle apresentaram um peso médio das penas de $58,25 \mathrm{~g}$. Nos animais intoxicados, a massa ficou reduzida em 33,81\% (38,55 g). A literatura sobre a influência das aflatoxinas no empenamento de aves ainda é muito restrita. No entanto, DOERR et al. (1983) realizaram pesquisas relacionadas ao tema sem constatarem efeito significativo na quantidade de folículos das penas de aves intoxicadas com ração contendo até $2,7 \mathrm{mg} / \mathrm{kg}$, durante os primeiros 42 dias de vida. Ao analisarmos a quantidade de aminoácidos totais contidos nas penas, observamos uma tendência de aumento na concentração dos mesmos nas penas das aves do grupo controle $(91,34 \mathrm{~g} / 100 \mathrm{~g})$ em relação ao grupo das aves intoxicadas $(91,15 \mathrm{~g} / 100 \mathrm{~g})$. No entanto, as médias não apresentaram diferença significativa quando analisadas pelo teste de Tukey $(\mathrm{P} \leq 0,10)$, inviabilizando o uso desta avaliação como critério para o diagnóstico da intoxicação em aves.

Ao avaliarmos o peso relativo de órgãos e carcaças, constatamos que o coração e o fígado das aves intoxicadas, apresentavam um aumento significativo de peso $(\mathrm{P} \leq 0,10)$. $\mathrm{O}$ baço e a moela, coletadas durante as necrópsias realizadas ao final do experimento (42 dias), não apresentaram diferenças significativas do peso relativo. $\mathrm{O}$ peso das carcaças das aves intoxicadas apresentaram uma redução significativa (Tabela 2). ORTATALI et al. (2004) intoxicaram frangos de corte durante 42 dias com quantidades inferiores de aflatoxinas $(50 \mu \mathrm{g}$ e $100 \mu \mathrm{g}$ de aflatoxina/kg de ração) e não observaram diferenças nos pesos relativos do fígado, rins, baço, timo e bursa de Fabricius, provavelmente devido à baixa dose de toxina utilizada. Esta tendência pode ser observada em outras espécies. HARVEY et al. (1989) intoxicaram experimentalmente 5 suínos com aflatoxinas $(0,75 \mathrm{mg} /$ $\mathrm{kg}$ de ração) e deoxinivalenol $(2,5 \mathrm{mg} / \mathrm{kg}$ de ração) e não observaram diferença significativa no peso relativo 
Tabela 1 - Consumo de ração e ganho de peso nas diferentes fases de criação de frangos de corte alimentados ad libitum com dietas tratadas ou não com aflatoxinas ( $3 \mathrm{mg} / \mathrm{kg}$ de ração) durante o período de 42 dias.

\begin{tabular}{lllllllllllll}
\hline \multirow{2}{*}{ Tratamento } & \multicolumn{3}{c}{$1-21$ dias } & \multicolumn{3}{c}{$22-35$ dias } & \multicolumn{3}{c}{$36-42$ dias } & \multicolumn{2}{c}{$1-42$ dias } \\
\cline { 2 - 11 } & Cons $(\mathrm{kg})$ & $\mathrm{GP}(\mathrm{kg})$ & $\mathrm{CA}$ & $\mathrm{Cons}(\mathrm{kg})$ & $\mathrm{GP}(\mathrm{kg})$ & $\mathrm{CA}$ & $\mathrm{Cons}(\mathrm{kg})$ & $\mathrm{GP}(\mathrm{kg})$ & $\mathrm{CA}$ & $\mathrm{Cons}(\mathrm{kg})$ & $\mathrm{GP}(\mathrm{kg})$ & $\mathrm{CA}$ \\
\hline $\mathrm{A}$ & $1,22^{\mathrm{a}}$ & $0,81^{\mathrm{a}}$ & $1,51^{\mathrm{a}}$ & $1,82^{\mathrm{a}}$ & $1,11^{\mathrm{a}}$ & $1,64^{\mathrm{a}}$ & $0,94^{\mathrm{a}}$ & $0,54^{\mathrm{a}}$ & $1,74^{\mathrm{a}}$ & $3,98^{\mathrm{a}}$ & $2,45^{\mathrm{a}}$ & $1,62^{\mathrm{a}}$ \\
& $(0,08)^{*}$ & $(0,05)$ & $(0,03)$ & $(0,11)$ & $(0,08)$ & $(0,06)$ & $(0,07)$ & $(0,02)$ & $(0,13)$ & $(0,21)$ & $(0,08)$ & $(0,04)$ \\
$\mathrm{B}$ & $0,77^{\mathrm{b}}$ & $0,51^{\mathrm{b}}$ & $1,50^{\mathrm{a}}$ & $1,61^{\mathrm{b}}$ & $0,89^{\mathrm{b}}$ & $1,81^{\mathrm{b}}$ & $0,49^{\mathrm{b}}$ & $0,40^{\mathrm{b}}$ & $1,24^{\mathrm{b}}$ & $2,86^{\mathrm{b}}$ & $1,79^{\mathrm{b}}$ & $1,59^{\mathrm{a}}$ \\
& $(0,03)$ & $(0,02)$ & $(0,05)$ & $(0,08)$ & $(0,06)$ & $(0,12)$ & $(0,03)$ & $(0,02)$ & $(0,11)$ & $(0,21)$ & $(0,11)$ & $(0,06)$ \\
\hline
\end{tabular}

a-b - Letras diferentes na mesma coluna representam diferença estatistica significativa entre os tratamentos comparados pelo teste de Tukey (P $\leq 0,10)$.

* - Desvio padrão

$\mathrm{A}=0 \mathrm{mg}$ de aflatoxina $/ \mathrm{kg}$ de ração; $\mathrm{B}=3 \mathrm{mg}$ de aflatoxina $/ \mathrm{kg}$ de ração

Cons $=$ Consumo $; \mathrm{GP}=$ Ganho de Peso $; \mathrm{CA}=$ Conversão Alimentar

de fígado, rins, baço e coração, quando comparados ao grupo controle.

As necropsias das aves que morreram durante, bem como ao final do experimento, evidenciaram alterações macroscópicas restritas aos animais do tratamento B (3mg de Aflatoxinas $/ \mathrm{kg}$ de ração). O fígado apresentou bordos arredondados, aumentados de tamanho e de coloração amarelo bronzeado. A vesícula biliar estava repleta, enquanto baço e rins apresentavam-se aumentados de tamanho e de coloração pálida. A bursa apresentou-se edemaciada e hemorrágica. Foi ainda evidenciado aumento no tempo de coagulação sanguínea no interior da cavidade abdominal, fato também constatado pelas hemorragias subcutâneas e musculares, principalmente no músculo tensor da fascia lata. Estas características também foram observadas por WYLLIE \& MOREHOUSE (1978), quando intoxicaram aves com ração contendo $100 \mu \mathrm{g}$ de Aflatoxinas $/ \mathrm{kg}$ de ração e observaram que as lesões ocorreram principalmente no fígado, que se apresentava pálido e ocasionalmente com pontos focais brancos e petéquiais, enquanto as vesículas biliares e ductos biliares apresentavam-se distendidas.
As alterações histopatológicas também foram observadas somente nas aves do tratamento B. No fígado, verificou-se degeneração hepática, com reação proliferativa ductal e hiperplásica dos ductos biliares. Nas bursas, foram encontradas vastas extensões da medular dos folículos com depleção linfóide, e nos rins, hemorragias multifocais e intensa degeneração gordurosa na forma de microvacuolização em células de alguns túbulos. Lesões similares também já foram descritas em aves intoxicadas com doses inferiores às utilizadas em nosso estudo. Frangos de corte intoxicados com $100 \mu \mathrm{g}$ de aflatoxinas $/ \mathrm{kg}$ de ração apresentaram ao final de 42 dias, significativas lesões hepáticas $(\mathrm{P}<0,05)$, quando comparados com um grupo controle, sendo que as principais alterações encontradas caracterizaram-se por degeneração hidrófica e vacúolos de gordura nos hepatócitos centrolobulares (ORTATALI et al., 2004).

Os resultados das análises sorológicas referentes à concentração das proteínas plasmáticas, permitiram evidenciar ao final do experimento, redução significativa na concentração das globulinas nas aves intoxicadas $(0,76 \mathrm{~g} / \mathrm{dL})$ em relação ao grupo controle $(1,52 \mathrm{~g} / \mathrm{dL})$. A concentração média de albumina foi de

Tabela 2 -Peso relativo dos órgãos de frangos de corte alimentados com dietas tratadas ou não com aflatoxinas (3mg / kg de ração), por um período de 42 dias.

\begin{tabular}{llllll}
\hline Tratamentos & Moela(\%) & Coração(\%) & Baço(\%) & Fígado(\%) & Carcaça(\%) \\
\hline A & $1,65^{\mathrm{a}}(0,29)^{*}$ & $0,59^{\mathrm{a}}(0,12)$ & $0,16^{\mathrm{a}}(0,13)$ & $2,42^{\mathrm{a}}(0,41)$ & $82,00^{\mathrm{b}}(0,15)$ \\
B & $1,95^{\mathrm{a}}(0,21)$ & $0,69^{\mathrm{b}}(0,11)$ & $0,19^{\mathrm{a}}(0,08)$ & $2,71^{\mathrm{b}}(0,42)$ & $80,19^{\mathrm{a}}(0,13)$ \\
\hline
\end{tabular}

a-b - Letras diferentes na mesma coluna representam diferença estatistica significativa entre os tratamentos comparados pelo teste de Tukey (P $\leq 0,10)$.

* - Desvio padrão

$\mathrm{A}=0 \mathrm{mg}$ de aflatoxina $/ \mathrm{kg}$ de ração $\mathrm{B}=3 \mathrm{mg}$ de aflatoxina $/ \mathrm{kg}$ de ração 
$1,67 \mathrm{~g} / \mathrm{dL}$ e $1,71 \mathrm{~g} / \mathrm{dL}$ para os tratamentos A e B, respectivamente, nao havendo diferença significante entre os grupos. A concentração das proteínas séricas das aves do tratamento A foi de $3,22 \mathrm{~g} / \mathrm{dL}$ e de $2,48 \mathrm{~g} / \mathrm{dL}$ para as aves do tratamento B. De acordo com as constatações de QUEZADA et al. (2000), frangos de corte tratados com $2,0 \mathrm{mg} / \mathrm{kg}$ de aflatoxinas apresentaram significante decréscimo $(\mathrm{P}<0,10)$ das proteínas plasmáticas (55\%) e albumina (80\%).

\section{CONCLUSÕES}

Frangos alimentados ad libitum com ração contendo $3 \mathrm{mg}$ de aflatoxinas/kg de ração apresentaram diminuição de consumo, ganho de peso e desuniformidade da estatura. Os órgãos mais atingidos pela intoxicação foram o fígado e o coração, bem como a carcaça das aves, cujo peso foi significativamente diminuído pela intoxicação. O peso das penas nas aves intoxicadas foi significativamente afetado, porém a concentração dos aminoácidos não apresentou alteração significativa, não permitindo a utilização deste parâmetro, como ferramenta de diagnóstico. A análise de aflatoxinas em alimentos de aves, segundo MALLMANN (2000) e detecção de resíduos metabólicos de aflatoxinas em tecidos animais (TRUCKSESS \& STOLOFF, 1981) são ferramentas importantes para diagnóstico de intoxicação por aflatoxinas em animais. Como diagnóstico presuntivo poderão ser utilizadas as avaliações clínicas das aves e as análises de lesões micro e macroscópicas.

\section{REFERÊNCIAS}

BONDINE, A.B.; MERTENS, L. Toxicology, metabolism and physiological effects of aflatoxin in the chicken. In. DIENER, U.L. et al. (Eds). Aflatoxin and Aspergillus flavus in corn. Auburn, Alabama: Auburn University, 1983. p.46-50. (Southern Cooperative Series Bulletin 279).

CELIK, I. et al. Efficacy of polyvinylpolypyrrolidone in reducing the immunotoxicity of aflatoxin in growing broilers. Poultry Science, v.41, n.4, p.430-439, 2000.

DILKIN, P. et al. Robotic automated clean-up for detection of fumonisins B1 and B2 in corn and corn-based feed by highperformance liquid chromatography. Journal of Chromatography - A, Irlanda, v.925, n.1/2, p.151-157, 2001.

DOERR, J.A. et al. Effects of low level chronic aflatoxicosis in broiler chickens. Poultry Science, v.62, n.10, p.1971-1977, 1983.

FERNANDEZ, A. et al. Changes in the prothrombin time, haematology and serum proteins during experimental aflatoxicosis in hens and broiler chickens. Research in Veterinary Science. n.58, p.119-122, 1995.
GHOSH, R.C. et al. Immunosupression in broiler under experimental aflatoxicosis. Br Vet J, v.146, p.457-462, 1990.

HUFF, W.E. et al. Progression of aflatoxicosis in broiler chickens. Poultry Science, v.65, p.1981-1989, 1986.

HARVEY, R.B. et al. Effects of aflatoxin, deoxynivalenol, and their combinations in the diets of growing pigs. American Journal Veterinary Research, v.50, n.4, p.602-607, 1989.

KICHOU, F.; WALSER, M.M. The natural occurrence of aflatoxin B1 in poultry feeds. Vet Hum Toxicol, v.35, n.2, p.105-108, 1993.

KUBENA, L.F. et al. Diminution of aflatoxicosis in growing chickens by the dietary addition of a hydrated sodium calcium aluminosilicate. Poultry Science, v.69, p.727-735, 1990.

LUNA, L.G. (Manual of histologic staining methods of the armed forces institute of pathology). 3.ed. New York: McGraw-Hill, 1968. Cap.3, p.68-71.

MALLMANN, C.A. et al. Automation of the analytical procedure for the simultaneous determination of aflatoxins $\mathrm{AFB}_{1}, \mathrm{AFB}_{2}, \mathrm{AFG}_{1}$ and $\mathrm{AFG}_{2}$ In: $\mathbf{X}$ INTERNATIONAL IUPAC SYMPOSIUM ON MYCOTOXINS AND PHYCOTOXINS. Guarujá, São Paulo, 2000. p.35.

MARIN, D.E. et al. Changes in performance, blood parameters, humoral and cellular immune responses in weanling piglets exposed to low doses of aflatoxin. Rev American Society of Animal Science, v.80, n.5, p.1250-1257, 2002.

NRC. Nutrient requirements of poultry. 9.ed. Washington: National Academy, 1994. 155p.

NURYONO, N. A limited survey of zearalenone in Indonesian maize-based food and feed by ELISA and high performance liquid chromatography. Food Control, v.16, n.1, p.65-71, 2004.

OLIVEIRA, C.; GERMANO, P. Aflatoxinas: conceitos sobre mecanismos de toxicidade e seu envolvimento na etiologia do câncer hepático celular. Rev Saúde Pública, v.31, n.4, p.417424, 1997.

ORGANIZACIÓN MUNDIAL DE LA SALUD. Criterios de salud ambiental - 11: Micotoxinas. Cidade do México: OPS, 1983. p.131.

ORTATALI, M. et al. Evaluation of pathological changes in broilers during chronic aflatoxin $(50$ and $100 \mathrm{ppb})$ and clinoptilolite exposure. Research in Veterinary Science, v.78, p.61-68, 2004.

PETSKA, J.J.; BONDY, G.S. Alteration of immune function following mycotoxin exposure. Can J Physiol Pharmacol, v.68, p.1009-1016, 1990.

PIER, A.C. The influence of mycotoxins on the immune system. In SMITH, J.E.; HENDERSON, R.S. Mycotoxins and animal foods. Boca Raton: CRC, 1991. p.875. 
PIER, A.C. Major biological consequences of aflatoxicosis in animal production. J Anim Sci, v.70, p.3964-3967, 1992

QUEZADA, T. et al. Effects of Aflatoxin $\mathrm{B}_{1}$ on the liver and kidney of broiler chickens during development. Comparative Biochemistry and Physiology, v.125, p.265-272, 2000.

RODRIGUES, E.F. Electro ionization mass spectral fragmentation of deoxinivalenol and related tricothecenes. Rapid Commun Mass Spectrom, v.16, p.1-9, 2002.

SHOTWEL, O.L. et al. Production of aflatoxin on rice. American Society for Microbiology, Michigan, USA, v.14, n.3, p.428-429, 1966 .
TANAKA, M.A.S. et al. Microflora fúngica de sementes de milho em ambientes de armazenamento. Sci Agric v.58, n.3, p.501-508, 2001 .

TRUCKESS, M.W; STOLOFF, L. High performance liquid chromatographic determination of aflatoxicol in milk, blood and liver. J Assoc off Anal Chem, v.54, n.5, p.1083-1087, 1981.

WYLLIE, T.D.; MOREHOUSE, L.G. Mycotoxicoses of domestic and laboratory animals, poultry, and aquatic invertebrates and vertebrates. Mycotoxic fungi, mycotoxins, micotoxicoses. Nova York: Marcel Dekker, 1978. V.2, p. 128 . 